\title{
Targeting Antibodies to Carbon Nanotube Field Effect Transistors by Pyrene Hydrazide Modification of Heavy Chain Carbohydrates
}

\author{
Steingrimur Stefansson, ${ }^{1}$ Hena H. Kwon, ${ }^{2}$ and Saeyoung Nate Ahn ${ }^{1}$ \\ ${ }^{1}$ Fuzbien Technology Institute, 9700 Great Seneca Hwy, Rockville, MD 20850, USA \\ ${ }^{2}$ MediSourcePlus, 222-22 Gurodong, Gurogu Seoul 336-708, Republic of Korea \\ Correspondence should be addressed to Steingrimur Stefansson, stennistef@fuzbien.com
}

Received 8 March 2012; Accepted 8 May 2012

Academic Editor: A. M. Rao

Copyright (C) 2012 Steingrimur Stefansson et al. This is an open access article distributed under the Creative Commons Attribution License, which permits unrestricted use, distribution, and reproduction in any medium, provided the original work is properly cited.

\begin{abstract}
Many carbon nanotube field-effect transistor (CNT-FET) studies have used immobilized antibodies as the ligand binding moiety. However, antibodies are not optimal for CNT-FET detection due to their large size and charge. Their size can prevent ligands from reaching within the Debye length of the CNTs and a layer of charged antibodies on the circuits can drown out any ligand signal. In an attempt to minimize the antibody footprint on CNT-FETs, we examined whether pyrene hydrazide modification of antibody carbohydrates could reduce the concentration required to functionalize CNT circuits. The carbohydrates are almost exclusively on the antibody Fc region and this site-specific modification could mediate uniform antibody orientation on the CNTs. We compared the hydrazide modification of anti-E. coli O157:H7 polyclonal antibodies to pyrenebutanoic acid succinimidyl ester-coated CNTs and carbodiimide-mediated antibody CNT attachment. Our results show that the pyrene hydrazide modification was superior to those methods with respect to bacteria detection and less than $1 \mathrm{nM}$ labeled antibody was required to functionalize the circuits.
\end{abstract}

\section{Introduction}

Label-free and real-time detection technologies circumvent the need for labeled detector molecules and optical-based detectors which, in turn, can decrease the time and cost of many clinical assays. Rapid, cost-effective, and realtime biomarker measurements are essential steps toward realizing the goal of personalized medicine. Detection of biomolecules based on their inherent properties, such as charge, can increase the speed and sensitivity of many biological assays. This can be achieved through field-effect transistors, including carbon nanotube field effect transistors (CNT-FET). Single-walled carbon nanotubes (SWCNTs) are nanomaterials that have great potential for the development of superior electronic instrumentation and detectors for biomedical applications $[1,2]$. SWCNTs are essentially rolled up graphite sheets and have excellent semiconductor properties but they retain the polyaromatic sp2 graphite bonds, which make them very chemically inert [3-5]. This property of CNTs limits the options available for covalently attaching biological agents on their surface, although CNT preparations can contain carboxyl groups at their termini and in sidewall defects [6] that can be utilized to covalently attach amine containing compounds through carbodiimide chemistry. More commonly, strong oxidants such as sulfuric acid/nitric acid mixtures are used to generate carboxyl groups on CNTs for that purpose $[7,8]$.

Noncovalent attachment is the preferred way to immobilize biologicals to CNTs because it is a relatively easy procedure and is performed under mild conditions, which is less likely to damage the CNTs or the biologicals being attached. 1-Pyrenebutyric acid N-hydroxysuccinimide ester (pyrene-NHS) has emerged as the reagent of choice for attaching a wide spectrum of molecules, including antibodies and aptamers to CNT-FETs [9-12]. Pyrenes are hydrophobic polycyclic aromatics that bind avidly to the hydrophobic CNTs and they do not adversely affect the electrical properties of the CNTs sp2 bonds [9]. The hydrophobic pyrene group anchors the reactive NHS ester to the nanotubes and allows it to react with solution phase molecules.

In practice, saturating concentrations of pyrene-NHS are added to the CNTs and allowed to adsorb, followed 
by removal of excess unbound reagent [9]. Using reactive pyrenes for immobilizing biological molecules onto CNTs is a convenient procedure since pyrene compounds with a range of reactive functional groups are readily available from many commercial vendors. The drawback to this procedure is that there is little control of the amount of pyreneNHS adhering to the CNTs. Additionally, NHS esters have a short half-life in aqueous solutions at physiological $\mathrm{pH}$ and temperature. Therefore, high concentrations of protein need to be used for this step to maximize the NHS ester reaction with amines because the competing reaction is alkaline hydrolysis [13]. This procedure can, therefore, lead to antibody saturation of the CNT's that can mask the signal of the bound ligand or decrease the ligand signal by sterically blocking them from approaching close enough to the CNT surface to influence the current.

We have developed and manufactured an inexpensive CNT-FET biosensor made on $4^{\prime \prime}$ standard semiconductor wafers containing 92 independent CNT-FET circuits [14]. We have used this system to measure serum insulin-like growth factor-1 (IGF-1) in a preclinical mouse model of human Brca1-related breast cancer using immobilized antibodies [14]. Recently, we expanded the repertoire of the CNT-FETs by using small hydrophobic boronic acid compounds to measure glycated human serum albumin, which is a marker for diabetes [15]. One of our goals is to develop this technology to measure multiple biomarkers in real time and to reach these goals we need to adopt and utilize established methodologies, including antibody-based detection. To date, many studies using CNT-FET-based detection have abandoned antibodies in lieu of aptamers, which are small synthetic nucleotide compounds that are selected for binding specific targets. Aptamers have, in some cases, demonstrated superior sensitivities $[11,16,17]$. However, aptamer technologies are yet not as mature and well established as antibody-based immunodetection with respect to specificity, availability, and cost. The ubiquity of antibodies in most biological detection assay makes their use all but unavoidable. Therefore, we are attempting approaches to functionalize CNT-FETs with antibodies by using sitespecific labeling that decreases antibody concentrations required to functionalize CNTs and thus minimizes their "footprint" on the circuits.

Most classes of antibodies contain highly conserved glycosylation sites. The carbohydrates have been proposed to increase their stability and also affect their biological activity with respect to complement activation. Mammalian IgGs have a single highly conserved glycosylation site (Asn297), which is located approximately in the middle of the heavy chain below the disulfide hinge region $[18,19]$. The oligosaccharides attached to $\operatorname{IgG}$ are $\sim 2 \mathrm{kDa}$ in size and consist mainly of galactose, mannose, $\mathrm{N}$-acetylglucosamine and terminating in sialic acid [20]. Despite their mass, the asparagine-glycosidic linkage is relatively flexible and the carbohydrates are often used to immobilize antibodies to a solid support because the conjugation does not significantly affect their antigen-binding capacity $[19,21,22]$.
In order to determine whether modifying antibody carbohydrates with pyrene offers an advantage for functionalizing CNT-FETs, we directly compared pyrene hydrazide modified anti-E. coli O157:H7 polyclonal antibodies to 2 previously published procedures; pyrene-NHS-coated CNTs and covalent carbodiimide attachment of antibodies. Our study shows that the pyrene-carbohydrate modification was superior to direct attachment of the antibody to CNTs via 1-Ethyl-3-(3-dimethylaminopropyl) carbodiimide hydrochloride (EDC) and the pyrene-NHS-coating method with respect to sensitivity and signal strength. Furthermore, the pyrene hydrazide reduces the concentration of antibody required to achieve optimal binding by about 100 folds.

\section{Materials and Methods}

2.1. Materials. Single-walled carbon nanotubes (SWCNTs) containing about $70 \%$ conducting nanotubes were purchased from Carbon Nanotechnologies Inc. 92 sample-well CNT-FETs were manufactured on standard $4^{\prime \prime}$ silica semiconductor wafers by NanoPlatform Inc. using standard photolithography and lift-off process as described by Kim et al. [23]. Briefly, an octadecyltrichlorosilane monolayer pattern on silicon oxide wafers was generated by first patterning AZ 5214 photoresist via standard photolithography, dipping the wafer in the octadecyltrichlorosilane solution $(1: 500, \mathrm{v} / \mathrm{v}$ in hexane) for $3 \mathrm{~min}$, and removing the PR patterns using acetone. The SWCNT solution was prepared by dispersing purified SWNTs in 1,2-dichlorobenzene with ultrasonication for $1 \mathrm{~h}$ (concentration $\sim 0.1 \mathrm{mg} / \mathrm{mL}$ ). Then, the patterned silicon oxide wafer was dipped in the SWCNT solution for $10 \mathrm{~s}$, rinsed thoroughly with 1,2-dichlorobenzene, and dried with nitrogen gas. This step allowed SWNTs to be adsorbed selectively onto bare $\mathrm{SiO}_{2}$ regions on the wafer, while the octadecyltrichlorosilane blocked nonspecific adsorption of the CNTs. After assembly of the SWCNTs, electrodes (30$\mathrm{nm}$ Au layer on 10-nm Pd) were fabricated via standard photolithography and a lift-off process.

Heat killed E. coli O157:H7, and anti-E. coli O157:H7 polyclonal antibodies were purchased from Kirkegaard and Perry Laboratories Inc. (Gaithersburg, MD, USA). A stock solution of $10^{9}$ colony forming units $/ \mathrm{mL}(\mathrm{CFU} / \mathrm{mL})$ E. coli O157: $\mathrm{H} 7$ was made using $\mathrm{diH}_{2} \mathrm{O}$. A $1 \mathrm{mg} / \mathrm{mL}$ stock solution of the antibody was made in $\mathrm{diH}_{2} \mathrm{O}$. Dimethyl formamide (DMF), 1-pyrene butanoic acid, 1-Pyrenebutyric acid Nhydroxysuccinimide ester, 1-pyrene butanoic acid hydrazide, 1-Ethyl-3-(3-dimethylaminopropyl) carbodiimide hydrochloride (EDC), N-Hydroxysuccinimide, sodium metaperiodate $\left(\mathrm{NaIO}_{4}\right), 2$-(N-morpholino)ethanesulfonic acid (MES), dimethyl sulfoxide (DMSO), and ethanol were purchased from VWR. GlycoLink Immobilization Kit and Zeba desalting columns were purchased from Pierce. All chemicals were used as received.

2.2. Attaching Anti-E. coli O157:H7 Antibodies to Carbon Nanotube Field Effect Transistors. In all cases, solutions were incubated on the wafers in closed containers to prevent 
evaporation and drying of antibodies and reagents onto the circuits.

The covalent attachment of antibodies to CNT carboxyl groups was performed using a two-step procedure involving activation of carboxyl groups by EDC followed by forming an amine reactive succinimide ester as previously described $[24,25]$, with minor modifications. Briefly, CNT-FET wafers were immersed in a $0.05 \mathrm{M}$ MES buffer $\mathrm{pH} 5.5$, containing $2 \mathrm{mM}$ EDC and $5 \mathrm{mM} \mathrm{N}$-hydroxysuccinimide and allowed to react for $15 \mathrm{~min}$ at $\mathrm{RT}$ after which wafers were quickly rinsed with $\mathrm{diH}_{2} \mathrm{O}$ and $5 \mu \mathrm{L}$ of $10 \mu \mathrm{g} / \mathrm{mL}$ of the polyclonal anti-E. coli O157:H7 antibody in PBS was incubated with the circuits for $1 \mathrm{hr}$ at RT. After incubation, the wafers were washed with $\mathrm{diH}_{2} \mathrm{O}$ to remove excess reagent and then air dried at RT prior to use.

Attachment of antibodies onto CNT-FET circuits via 1-pyrenebutyric acid N-hydroxysuccinimide ester (pyreneNHS) was performed essentially as previously described [9, 26], with minor modifications. Briefly, wafers were immersed in a $5 \mathrm{mM}$ solution of pyrene-NHS in ethanol with gentle agitation for $1 \mathrm{hr}$ at RT. After the incubation, wafers were quickly rinsed with ethanol and $5 \mu \mathrm{L}$ of $10 \mu \mathrm{g} / \mathrm{mL}$ of the polyclonal antibody against E. coli O157:H7 in PBS was incubated with the circuits for $1 \mathrm{hr}$ at RT. Wafers were then washed with $\mathrm{diH}_{2} \mathrm{O}$ and dried. A modification of this procedure, which involves incubating wafers with a mixture of pyrene butanoic acid and pyrene-NHS was also performed as previously described $[14,23]$, with minor modification. Briefly, CNT-FET wafer circuits were incubated with $10 \mu \mathrm{L}$ of a $5 \mathrm{mM}$ solution of 1-pyrene butanoic acid and pyrene-NHS in DMF at a molar ration of $3: 1$, respectively, for $30 \mathrm{~min}$ at RT. After the incubation, wafers were briefly washed with ethanol and dried. $5 \mu \mathrm{L}$ of the anti-E. coli O157:H7 antibody at $10 \mu \mathrm{g} / \mathrm{mL}$ in PBS was added to the circuits and incubated for $1 \mathrm{hr}$ at RT after which the wafers were washed with $\mathrm{diH}_{2} \mathrm{O}$ and dried.

Attaching 1-pyrenebutanoic acid hydrazide to antibody carbohydrates was performed using a procedure described by Hermanson [27], with minor modification. Briefly, $0.6 \mathrm{mg}$ of the anti-E. coli O157:H7 antibody in PBS was made $10 \mathrm{mM}$ in $\mathrm{NaIO}_{4}$ and kept in the dark for $15 \mathrm{~min}$ at RT followed by removal of excess reagent by desalting column. 1-pyrene butanoic acid hydrazide (from DMSO stock) was added to the antibody solution for a final concentration of $20 \mu \mathrm{M}$ in and incubated for $2 \mathrm{hr}$ at RT, followed by removal of unreacted reagent by centrifugation and adding the sample to a desalting column. The extent of pyrene labeling was determined by measuring the absorbance of the labeled antibodies at $280 \mathrm{~nm}$ and $343 \mathrm{~nm}$. We used the molar extinction coefficients of $2.1 \times 10^{5} \mathrm{M}^{-1} \mathrm{~cm}^{-1}$ for IgG [28] and $2.3 \times 10^{4} \mathrm{M}^{-1} \mathrm{~cm}^{-1}$ for pyrene $[29,30]$. Additionally, we measured a solution of pyrene butanoic acid in PBS at $280 \mathrm{~nm}$ and $343 \mathrm{~nm}$ to obtain a correction factor (A280/A343) of 0.669. Using a formula provided by Pierce [28], we calculated the extent of pyrene labeling to be between 8-9 pyrenes per IgG, which correlates with values for polyclonal antibody labeling obtained by Wolfe and Hage [31].
To find the optimal coating concentration of the pyrenehydrazide labeled antibody, the antibody was titrated onto CNT-FET circuits. Briefly, $5 \mu \mathrm{L}$ of the pyrene hydrazide labeled antibodies in $\mathrm{diH}_{2} \mathrm{O}$ were incubated on CNT-FET circuits for $1 \mathrm{hr}$ at RT, after which the wafers were washed with $\mathrm{diH}_{2} \mathrm{O}$ and dried. Aliquots of the antibody were stored at $-80^{\circ} \mathrm{C}$.

\subsection{Measuring E. coli O157:H7 Binding to Carbon Nanotube} Field Effect Transistors. The 92 circuit CNT-FET wafers used for this study and their characteristics were previously described in Jones et al. [14]. For the bacteria detection assays, a baseline impedance value for each circuit was first obtained by applying $10 \mu \mathrm{L} \mathrm{diH}_{2} \mathrm{O}$ to the circuit and recording the signal for approximately $30 \mathrm{sec}$. Then, $10 \mu \mathrm{L}$ of the $E$. coli in $\operatorname{diH}_{2} \mathrm{O}$, at concentrations indicated in the text and figure legends, were carefully admixed with the $10 \mu \mathrm{L} \mathrm{diH}_{2} \mathrm{O}$ on the circuits and changes in impedance were measured for up to 3.5 additional minutes. The impedance value for each measurement was normalized to the corresponding baseline value. Each solution of the E. coli was measured at least in quadruplicate using a fresh circuit for each measurement. A source/drain bias of $100 \mathrm{mV}$ was maintained throughout the measurements of the electrical signal and the pulse width was $1 \mathrm{sec}$. The reference electrode is the back (bottom) side of the grounded wafer. The device uniformity was not optimized for entire wafers, but individual circuits used for the assays were carefully evaluated before experiments. The selected CNTFET circuits ranged typically between five and ten in on-off ratio. The electrical properties of the samples binding the CNT-FET were measured using a low current measurement system (MediSourcePlus Inc.) that makes electrical contact to the source and drain electrodes of the CNT-FET. The transfer characteristics of this circuit design were previously characterized for detection of prostate specific antigen and IGF-1 [14, 23]. Briefly, typical observed electronic transfer changes from 20 to 10 nanoamperes before and after the antibody immobilization on the CNT-FET circuits when $V_{\mathrm{ds}}$ and $V_{\mathrm{G}}$ are 0.1 and -0.1 volt, respectively. The response in the electrical signal is typically in the range of 2 to $20 \%$ in the normalized units.

\section{Results and Discussion}

Antibodies are the mainstay of many biological detection assays and are, therefore, the logical first choice to use as ligand capture molecules for CNT-FET. However, CNT-FET current is very sensitive to charges in the immediate environment and a full-length $\operatorname{IgG}(150 \mathrm{kDa})$ can contain about 90 glutamic/aspartic acids and around 100 lysines/arginines (Genebank: AAA51043.1 and CAA05417.1), most of which are ionized at physiological $\mathrm{pH}$. Therefore, immobilizing antibodies onto CNT-FETs will inevitably influence the current. Furthermore, compared to many other immunodetection methods, CNT-FETs are relatively unwieldy with respect to antibody functionalization. Unlike ELISA and many other immunodetection methods, the ligand itself 

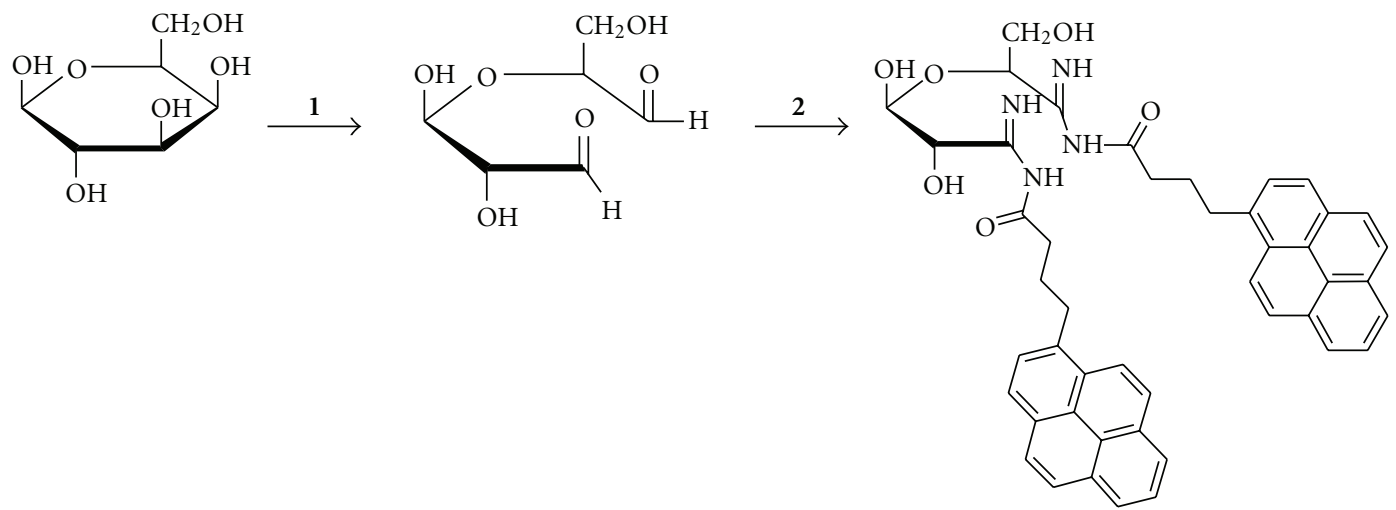

FIGURE 1: Reactions performed to modify antibody carbohydrates with pyrene hydrazide, illustrated with galactose. Step 1: antibodies are treated with $10 \mathrm{mM} \mathrm{NaIO}_{4}$, which oxidizes cis-diols to aldehyde groups. Step 2: after a desalting step, the antibody, it is reacted with 1pyrenebutanoic acid hydrazide which forms stable hydrazone bonds with the formed aldehyde groups.

generates the signal on the CNT-FETs, thus making the concentration of attached antibody a critical factor. High densities of antibodies on CNT-FETs can lessen the signal because the abundance of charged antibodies on the circuits can drown out any ligand charge. A layer of antibodies on the CNTs can also keep the ligand far enough from the Debye length to have any influence on the current. Conversely, low antibody densities on the CNT-FETs also decrease the signal [23]. The spatial orientation of the antibodies on the CNT's is also important for signal strength and consistency because of the limited number of antibodies that can occupy the small CNT-FET surface.

There are currently two strategies used to attach antibodies to CNTs. (i) covalent attachment of antibodies to the CNTs and (ii) noncovalent adsorption of antibodies either directly on to the CNTs or via hydrophobic compounds containing reactive groups $[32,33]$. In this study, we present a third possibility, namely, the specific labeling of antibody carbohydrate region with pyrene hydrazide. Labeling the carbohydrate groups has many practical purposes. (1) Most, if not all, IgG's contain a single highly conserved $\mathrm{N}$ glycosylation site in the Fc region of the heavy chains (Asn279), that does not overlap with the IgG variable regions (antigen binding sites). (2) Modifying this site will produce uniformly labeled antibodies that will likely bind CNT's in a uniform orientation and generate a more consistent signal.

(3) Labeling procedures and protocols are well established. (4) Most polyclonal and many monoclonal IgG's contain carbohydrate groups, although to a varying degree.

Figure 1 illustrates the reactions performed to modify antibody carbohydrates with pyrene hydrazide. Some carbohydrates such as $(\mathrm{D}+)$ galactose and mannose contain a planar pair of hydroxyl groups (cis-diols) that can be oxidized with mild reagents, such as sodium periodate, to generate aldehydes. Each IgG can have up to four galactose and six mannose residues per protein [19].

Shown in the figure is the structure of $\beta$-D-galactose, which has a cis-diol. Periodate oxidation opens up the ring structure by converting the cis-diols to aldehyde groups. At $10 \mathrm{mM}$, periodate will also generate an aldehyde group on terminal sialic acid residues present on most IgG carbohydrate regions $[19,34]$. Aldehyde functional groups are not found in natural amino acids [35]. They are very reactive at neutral $\mathrm{pH}$ and this allows for modifications with aldehydespecific reagents such as pyrene butanoic acid hydrazide. The products of the labeling are stable pyrene hydrazone derivatives attached to the antibody carbohydrate in the middle of the heavy chain Fc region $[19,36]$. Furthermore, since the labeling procedure generated about 8 pyrenes per antibody (see Section 2), we surmise that they will be equally distributed between the two heavy chains of the antibody and will, therefore, likely anchor the antibody to the CNTs in a uniform manner.

Figure 2 illustrates how the E. coli antibodies might orient on the CNT-FET circuits using the three different antibody immobilization procedures performed in this study. Figure 2(a) illustrates covalent attachment of antibodies to CNT-FETs by activating CNT carboxyl groups with EDC in the presence of N-hydroxysuccinimide, which will generate an NHS ester that reacts with primary amines. This 2-step procedure was used because coupling with EDC alone will also yield crosslinked antibody multimers because of intermolecular reactions between antibody amine and carboxyl groups [37]. Another reason for using Nhydroxysuccinimide is that the $\mathrm{O}$-acylisourea intermediate formed in the reaction of EDC with carboxylic acids is very unstable in aqueous solution and reduces the yield [37]. Regardless of whether EDC or EDC/N-hydroxysuccinimide is used, both strategies will yield the same product, namely antibodies directly attached to CNTs without any spacer groups. Since the antibody amine groups are randomly distributed on the protein, their orientation on the circuits will likely also be random. Figure 2(b) depicts attachment of antibodies to CNT-FETs via preadsorbed pyrene-NHS. The pyrene moiety will adhere to the CNTs, likely through $\pi-\pi$ interactions, leaving the NHS ester exposed to the solvent and accessible to proteins. The butanoic acid provides a four carbon spacer arm that allows the bound antibody some degree of freedom. The antibodies coupled using the pyrene-NHS will also likely be randomly oriented on 

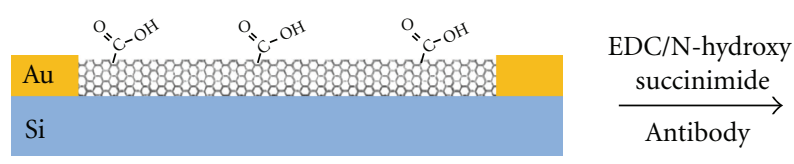

(a)
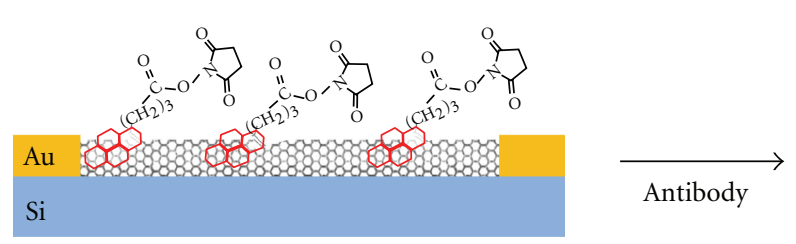

(b)

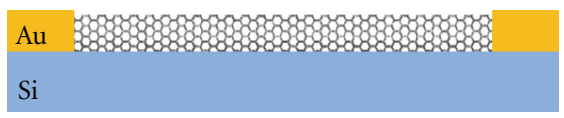

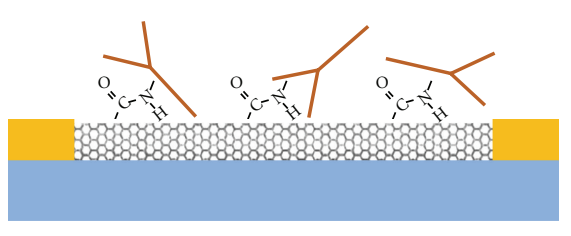
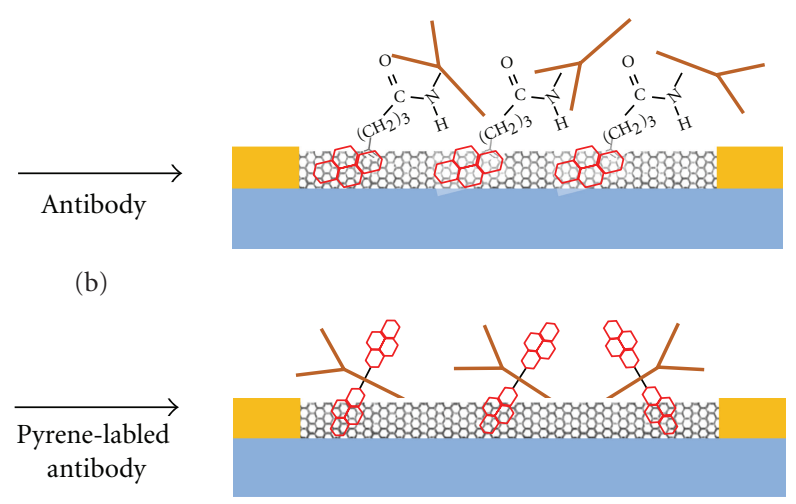

(c)

FIGURE 2: Depiction of the CNT-FET antibody functionalization procedures used in this study. (a) EDC/N-Hydroxy succinimide modification of endogenous CNT carboxyl groups (left). Using this method the antibody is attached to the CNTs via zero-length coupling (right). (b) Pyrenebutanoic acid succinimide esters addition to CNTs (left), which mediate attachment to antibody primary amine groups via a four carbon spacer (right). (c) Addition of pyrene-hydrazide modified antibodies to bare CNT-FETs (left), which can lead to uniform antibody orientation on the circuits (right).

the circuits because of the random distribution of antibody amine residues. Figure 2(c) illustrates coating CNT-FET circuits using pyrene-hydrazide-modified antibodies. The antibodies will likely orient on the circuits in a uniform manner because the hydrophobic pyrene residues will only be on the carbohydrate region of the antibodies.

To determine the optimal coating concentration for the pyrene hydrazide labeled E. coli O157:H7 antibodies, we titrated them onto CNT-FET circuits. The antibody was incubated with the circuits at the indicated concentrations shown in Figure 3. Following wash, $10^{7} \mathrm{cfu} / \mathrm{mL}$ of E. coli O157:H7 was added to the circuits and impedance was measured as described in Section 2. The results shown in Figure 3 demonstrate a bell-shaped response curve of $E$. coli binding to different concentrations of the antibody, with an optimum antibody coating concentration of around $0.5 \mathrm{nM}$. Previously, we observed a bell-shaped impedance curve in our study of binding of glycated human serum albumin to hydrophobic boronic acid compounds titrated onto CNT-FET circuits, demonstrating the importance of coating concentrations of the capture moieties for maximal ligand signal [15]. Additionally, a bell-shaped response was also observed titrating pyrene-hydrazide-labeled polyclonal antibodies to human IgG (data not shown). The reason for the observed bell shaped curves, in lieu of saturation binding, could be due to the limited surface area available on the CNT-FET circuits. With increasing coating concentrations the ligands could, therefore, be aggregating onto the CNTs and masking them from the target.

We compare the 3 different antibody coating procedures depicted in Figure 2 and described in Section 2 with respect

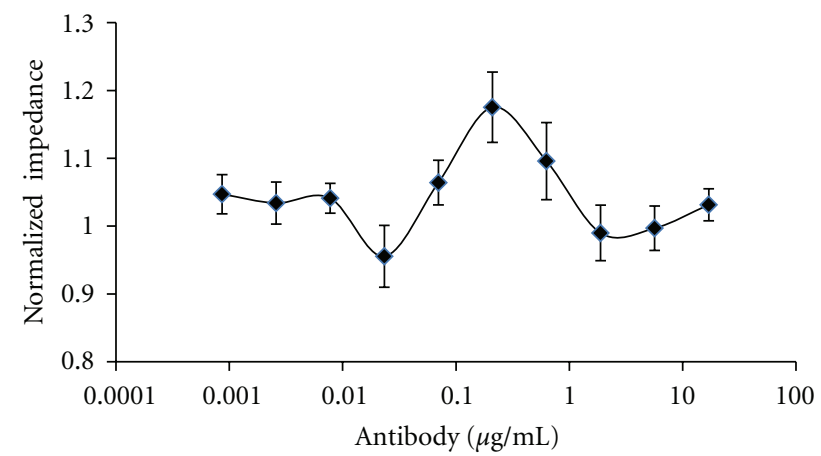

Figure 3: Determination of optimal coating concentration for pyrene-hydrazide labeled E. coli O157:H7 polyclonal antibody. CNT-FET circuits were coated with labeled antibody as described in Section 2 followed by addition of $10 \mu \mathrm{L}$ of $10^{7} \mathrm{cfu} / \mathrm{mL}$ E. coli O157:H7 in $\mathrm{diH}_{2} \mathrm{O}$. Impedance was measured for 4 minutes after addition of the bacteria and plotted as the normalized impedance compared to baseline value of the individual circuit. Each point represents at least 5 measurements with standard error of the mean.

to binding E. coli O157:H7. The responses of the circuits were measured by titrating E. coli $\mathrm{O} 157: \mathrm{H} 7$ in $\mathrm{diH}_{2} \mathrm{O}$ onto the circuits. The data presented in Figure 4 suggests that the targeted pyrene modification of the antibody carbohydrates coated at the optimal coating concentrations $(0.5 \mathrm{nM})$ yielded better results both in terms of signal strength and sensitivity compared to the EDC/NHS and NHS-pyrene procedures. The pyrene-hydrazide-modified antibodies will likely orient in a uniform manner on the circuits because the hydrophobic pyrene residues associating with the CNTs will 


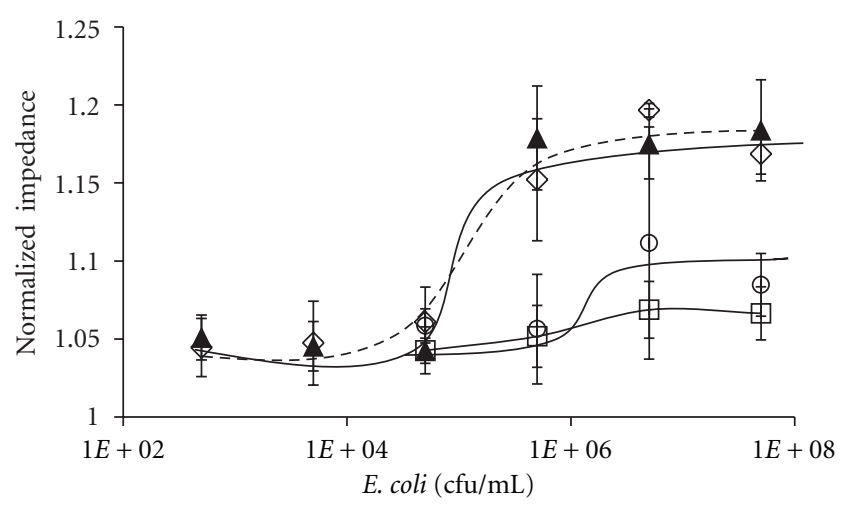

Figure 4: Comparison of CNT-FET antibody coating procedures. Increasing concentrations of E. coli O157:H7 were added to E. coli O157:H7 polyclonal antibody immobilized onto CNT-FET circuits using either EDC/NHS ( $\square$ ), Pyrenebutyric acid succinimide ester (०), Pyrene hydrazide modified antibody $(\boldsymbol{\Lambda})$ or pyrenebutanoic acid/ pyrenebutanoic acid succinimide ester at a molar ratio of $3: 1$ $(\diamond$, dotted line) as described in Section 2. Impedance was measured for 4 minutes after addition of the bacteria and plotted as the normalized value compared to the baseline of the individual circuit. Each point represents at least 5 measurements with standard error of the mean.

only be on the carbohydrate region of the antibodies. Since the carbohydrate region is in the middle of the Fc portion of the antibodies, the antigen binding sites might not be sterically hindered by the CNT surface. The other methods used lead to random orientation of antibodies on the circuits and thus decrease their effective concentration.

Pyrene-hydrazide labeled antibodies was able to detect around $10^{5} \mathrm{cfu} / \mathrm{mL}$ of E. coli O157:H7 cells, which is similar to that reported by Garcia-Aljaro et al. [38] using a response time of $60 \mathrm{~min}$. In our experiments, the impedance reached a plateau after 3-4 min. Higher sensitivity for E. coli O157:H7 on CNT-FET has been reported using aptamers made against the bacteria as the binding moiety [39]. Although the authors used a different attachment methodology from those reported here, the smaller size of the aptamer likely plays a role in the increased sensitivity.

The low signal obtained from the EDC/NHS method could suggests that there are few available carboxyl groups on the CNT termini available for antibody attachment, despite the CNT preparations being ultrasonicated prior to their deposition onto the wafers [23]. The ultrasonication shortens the CNTs and can cause the termini to react with environmental factors [40,41]. Some studies oxidize the CNTs prior to making the FET circuits to ensure that enough carboxyl functional groups are present prior to the carbodiimide coupling $[7,8]$. Alternatively, it is possible that coupling the antibodies directly onto CNTs without a spacer arm separating them, either limits their capability to interact with the antigen or destabilizes their structure, as has been shown for some proteins adsorbed onto CNTs [42, 43].

Preloading the CNT-FETs with pyrene-NHS did yield higher signal than the EDC/NHS procedure but was less sensitive than the targeted pyrene hydrazide modification.
One possibility for the observed difference in these two methodologies is that the pyrene-NHS procedure results in higher than optimal antibody concentrations on the CNTs. To address this possibility, we coated circuits according to procedure by Kim et al. [23]. Briefly, their study showed that coating CNT-FET circuits with a 3:1 molar ratio of inert pyrene butanoic acid and pyrene-NHS, instead of pyreneNHS alone, increased the sensitivity of the circuits when measuring prostate specific antigen (PSA) using a monoclonal antibody. They showed that the inert pyrene butanoic acid acts as a competitor to the pyrene-NHS binding to the CNTs. This effectively reduces the pyrene-NHS coating concentration and, according to the researchers, reduces the steric hindrance of antibodies on the circuits [23]. Our results in Figure 4 demonstrate that addition of pyrene butanoic acid improves the signal strength and sensitivity over that of using pyrene-NHS alone and supports the observations by Kim et al. [23]. Together these results highlight the importance that antibody coating concentrations have on CNT-FET performance. Coating the CNT circuits with a mixture of reactive and inert pyrenes, though, has significant drawbacks over that of coating pyrene-hydrazidelabeled antibodies directly onto the circuits. The reactive pyrene-NHS ester moiety is very unstable and great care must be taken to keep the product free of moisture before coating the circuits. After coating, antibodies need to be added immediately to the circuits and at high concentrations to ensure effective labeling. In contrast, around 100 -fold less pyrene-hydrazide antibody is needed for the CNT coating and the labeled antibodies are stable at $4^{\circ} \mathrm{C}$ for over 2 weeks or can be stored at $-80^{\circ} \mathrm{C}$ for extended periods.

\section{Conclusion}

In this study, we compare different methodologies of attaching antibodies to carbon nanotube field effect transistors. The two published methods used were covalent binding of antibodies to CNTs and attachment of antibodies to reactive pyrenes adsorbed to the circuits. We compared the efficacy of these methods to our procedure described herein, which is covalently attaching pyrene moieties to the carbohydrate residues of the antibodies prior to the addition to the CNT-FETs. The pyrene-hydrazide modification proved to be superior to the other methods, with respect to sensitivity and signal strength. We feel that the main advantage this procedure has is that antibody coating concentrations can be controlled. Also it is likely that the orientation of the antibodies on the circuits is more uniform. The hydrazide reaction can be performed in an afternoon and the concentrations of hydrazide-modified antibody required to coat the circuits are small. Furthermore, storing the labeled antibody at $-80^{\circ} \mathrm{C}$ will ensure that the same preparation can be used to coat the circuits, which will aid in generating more consistent results. However, it should be noted that this study was performed using polyclonal antibodies which, in most cases, possess fully mature glycosylation sites. Monoclonals, on the other hand, can be variable in their glycosylation. 


\section{Acknowledgments}

This work was supported in part by the Maryland Industrial Partnership (MIPS) program, no. 4319, and by the Maryland Technology Development Corporation's Maryland Technology Transfer and Commercialization Fund.

\section{References}

[1] P. Vichchulada, Q. Zhang, and M. D. Lay, "Recent progress in chemical detection with single-walled carbon nanotube networks," Analyst, vol. 132, no. 8, pp. 719-723, 2007.

[2] D. R. Kauffman and A. Star, "Electronically monitoring biological interactions with carbon nanotube field-effect transistors," Chemical Society Reviews, vol. 37, no. 6, pp. 11971206, 2008.

[3] C. Soldano, A. Mahmood, and E. Dujardin, "Production, properties and potential of graphene," Carbon, vol. 48, no. 8 , pp. 2127-2150, 2010.

[4] P. Avouris, "Molecular electronics with carbon nanotubes," Accounts of Chemical Research, vol. 35, no. 12, pp. 1026-1034, 2002.

[5] D. A. Britz and A. N. Khlobystov, "Noncovalent interactions of molecules with single walled carbon nanotubes," Chemical Society Reviews, vol. 35, no. 7, pp. 637-659, 2006.

[6] D. Tasis, N. Tagmatarchis, A. Bianco, and M. Prato, "Chemistry of carbon nanotubes," Chemical Reviews, vol. 106, no. 3, pp. 1105-1136, 2006.

[7] Y. Xiao, X. Gao, O. Taratula et al., "Anti-HER2 IgY antibodyfunctionalized single-walled carbon nanotubes for detection and selective destruction of breast cancer cells," BMC Cancer, vol. 9, p. 351, 2009.

[8] T. Ramanathan, F. T. Fisher, R. S. Ruoff, and L. C. Brinson, "Amino-fimctionalized carbon nanotubes for binding to polymers and biological systems," Chemistry of Materials, vol. 17, no. 6, pp. 1290-1295, 2005.

[9] R. J. Chen, Y. Zhang, D. Wang, and H. Dai, "Noncovalent sidewall functionalization of single-walled carbon nanotubes for protein immobilization," Journal of the American Chemical Society, vol. 123, no. 16, pp. 3838-3839, 2001.

[10] A. Star, J. C. P. Gabriel, K. Bradley, and G. Gruner, "Electronic detection of specific protein binding using nanotube FET devices," Nano Letters, vol. 3, no. 4, pp. 459-463, 2003.

[11] K. Maehashi, T. Katsura, K. Kerman, Y. Takamura, K. Matsumoto, and E. Tamiya, "Label-free protein biosensor based on aptamer-modified carbon nanotube field-effect transistors," Analytical Chemistry, vol. 79, no. 2, pp. 782-787, 2007.

[12] P. Hu, J. Zhang, L. Li, Z. Wang, W. O’Neill, and P. Estrela, "Carbon nanostructure-based field-effect transistors for labelfree chemical/biological sensors," Sensors, vol. 10, no. 5, pp. 5133-5159, 2010.

[13] G. T. Hermanson, Bioconjugate Techniques, Academic Press, San Diego, Calif, USA, 1996.

[14] L. P. Jones, S. Stefansson, M. Kim, and S. N. Ahn, "Comparison of radioimmuno and carbon nanotube field-effect transistor assays for measuring insulin-like growth factor-1 in a preclinical model of human breast cancer," Journal of Nanobiotechnology, vol. 9, article 36, 2011.

[15] S. Stefansson, L. A. Stefansson, S. W. Chung, K. Ko, H. H. Kwon, and S. N. Ahn, "Evaluation of aromatic boronic acids as ligands for measuring diabetes markers on carbon nanotube field-effect transistors," Journal of Nanotechnology, vol. 2012, Article ID 371487, 6 pages, 2012.
[16] A. Bini, M. Minunni, S. Tombelli, S. Centi, and M. Mascini, "Analytical performances of aptamer-based sensing for thrombin detection," Analytical Chemistry, vol. 79, no. 7, pp. 30163019, 2007.

[17] J. H. Lee, M. V. Yigit, D. Mazumdar, and Y. Lu, "Molecular diagnostic and drug delivery agents based on aptamernanomaterial conjugates," Advanced Drug Delivery Reviews, vol. 62, no. 6, pp. 592-605, 2010.

[18] M. Nose and H. Wigzell, "Biological significance of carbohydrate chains on monoclonal antibodies," Proceedings of the National Academy of Sciences of the United States of America, vol. 80, no. 21, pp. 6632-6636, 1983.

[19] J. N. Arnold, M. R. Wormald, R. B. Sim, P. M. Rudd, and R. A. Dwek, "The impact of glycosylation on the biological function and structure of human immunoglobulins," Annual Review of Immunology, vol. 25, pp. 21-50, 2007.

[20] R. Jefferis, J. Lund, H. Mizutani et al., "A comparative study of the N-linked oligosaccharide structures of human IgG subclass proteins," Biochemical Journal, vol. 268, no. 3, pp. 529-537, 1990.

[21] W. L. Hoffman and D. J. O'Shannessy, "Site-specific immobilization of antibodies by their oligosaccharide moieties to new hydrazide derivatized solid supports," Journal of Immunological Methods, vol. 112, no. 1, pp. 113-120, 1988.

[22] V. V. Shmanai, T. A. Nikolayeva, L. G. Vinokurova, and A. A. Litoshka, "Oriented antibody immobilization to polystyrene macrocarriers for immunoassay modified with hydrazide derivatives of poly(meth)acrylic acid," BMC Biotechnology, vol. 1, no. 1, p. 4, 2001.

[23] J. P. Kim, B. Y. Lee, J. Lee, S. Hong, and J. Sim, "Enhancement of sensitivity and specificity by surface modification of carbon nanotubes in diagnosis of prostate cancer based on carbon nanotube field effect transistors," Biosensors and Bioelectronics, vol. 24, no. 11, pp. 3372-3378, 2009.

[24] G. T. Hermanson, in Bioconjugate Techniques, pp. 170-177, Academic Press, San Diego, Calif, USA, 1996.

[25] K. Jiang, L. S. Schadler, R. W. Siegel, X. Zhang, H. Zhang, and M. Terrones, "Protein immobilization on carbon nanotubes via a two-step process of diimide-activated amidation," Journal of Materials Chemistry, vol. 14, no. 1, pp. 37-39, 2004.

[26] C. Li, M. Curreli, H. Lin et al., "Complementary detection of prostate-specific antigen using $\operatorname{In}_{2} \mathrm{O}_{3}$ nanowires and carbon nanotubes," Journal of the American Chemical Society, vol. 127, no. 36, pp. 12484-12485, 2005.

[27] G. T. Hermanson, in Bioconjugate Techniques, pp. 340-341, Academic Press, San Diego, Calif, USA, 1996.

[28] http://www.piercenet.com/instructions/2160087.pdf.

[29] A. C. Sen and B. Chakrabarti, "Proximity of sulfhydryl groups in lens proteins. Excimer fluorescence of pyrenelabeled crystallins," Journal of Biological Chemistry, vol. 265, no. 24, pp. 14277-14284, 1990.

[30] S. S. Lehrer, P. Graceffa, and D. Betteridge, "Conformational dynamics of tropomyosin in solution: evidence for two conformational states," Annals of the New York Academy of Sciences, vol. 366, pp. 285-299, 1981.

[31] C. A. Wolfe and D. S. Hage, "Studies on the rate and control of antibody oxidation by periodate," Analytical Biochemistry, vol. 231, no. 1, pp. 123-130, 1995.

[32] P. Singh, S. Campidelli, S. Giordani, D. Bonifazi, A. Bianco, and M. Prato, "Organic functionalisation and characterisation of single-walled carbon nanotubes," Chemical Society Reviews, vol. 38, no. 8, pp. 2214-2230, 2009.

[33] K. Yang and B. Xing, "Adsorption of organic compounds by carbon nanomaterials in aqueous phase: Polanyi theory and 
its application," Chemical Reviews, vol. 110, no. 10, pp. 5989$6008,2010$.

[34] G. T. Hermanson, in Bioconjugate Techniques, p. 108, Academic Press, San Diego, Calif, USA, 1996.

[35] P. Wu, W. Shui, B. L. Carlson et al., "Site-specific chemical modification of recombinant proteins produced in mammalian cells by using the genetically encoded aldehyde tag," Proceedings of the National Academy of Sciences of the United States of America, vol. 106, no. 9, pp. 3000-3005, 2009.

[36] G. T. Hermanson, in Bioconjugate Techniques, pp. 472-478, Academic Press, San Diego, Calif, USA, 1996.

[37] Y. Gao and I. Kyratzis, "Covalent immobilization of proteins on carbon nanotubes using the cross-linker 1-ethyl-3-(3dimethylaminopropyl)carbodiimide - a critical assessment," Bioconjugate Chemistry, vol. 19, no. 10, pp. 1945-1950, 2008.

[38] C. Garcia-Aljaro, L. N. Cella, D. J. Shirale et al., "Carbon nanotubes-based chemiresistive biosensors for detection of microorganisms," Biosensors and Bioelectronics, vol. 26, no. 4, pp. 1437-1441, 2010.

[39] H. M. So, D. W. Park, E. K. Jeon et al., "Detection and titer estimation of Escherichia coli using aptamer-functionalized single-walled carbon-nanotube field-effect transistors," Small, vol. 4, no. 2, pp. 197-201, 2008.

[40] A. Koshio, M. Yudasaka, M. Zhang, and S. Iijima, "A simple way to chemically react single-wall carbon nanotubes with organic materials using ultrasonication," Nano Letters, vol. 1, no. 7, pp. 361-363, 2001.

[41] J. Hilding, E. A. Grulke, Z. G. Zhang, and F. Lockwood, "Dispersion of carbon nanotubes in liquids," Journal of Dispersion Science and Technology, vol. 24, no. 1, pp. 1-41, 2003.

[42] S. S. Karajanagi, A. A. Vertegel, R. S. Kane, and J. S. Dordick, "Structure and function of enzymes adsorbed onto singlewalled carbon nanotubes," Langmuir, vol. 20, no. 26, pp. 11594-11599, 2004.

[43] S. F. Hsieh, D. Bello, D. F. Schmidt, A. K. Pal, and E. J. Rogers, "Biological oxidative damage by carbon nanotubes: fingerprint or footprint?" Nanotoxicology, vol. 6, no. 1, pp. 6176, 2012. 

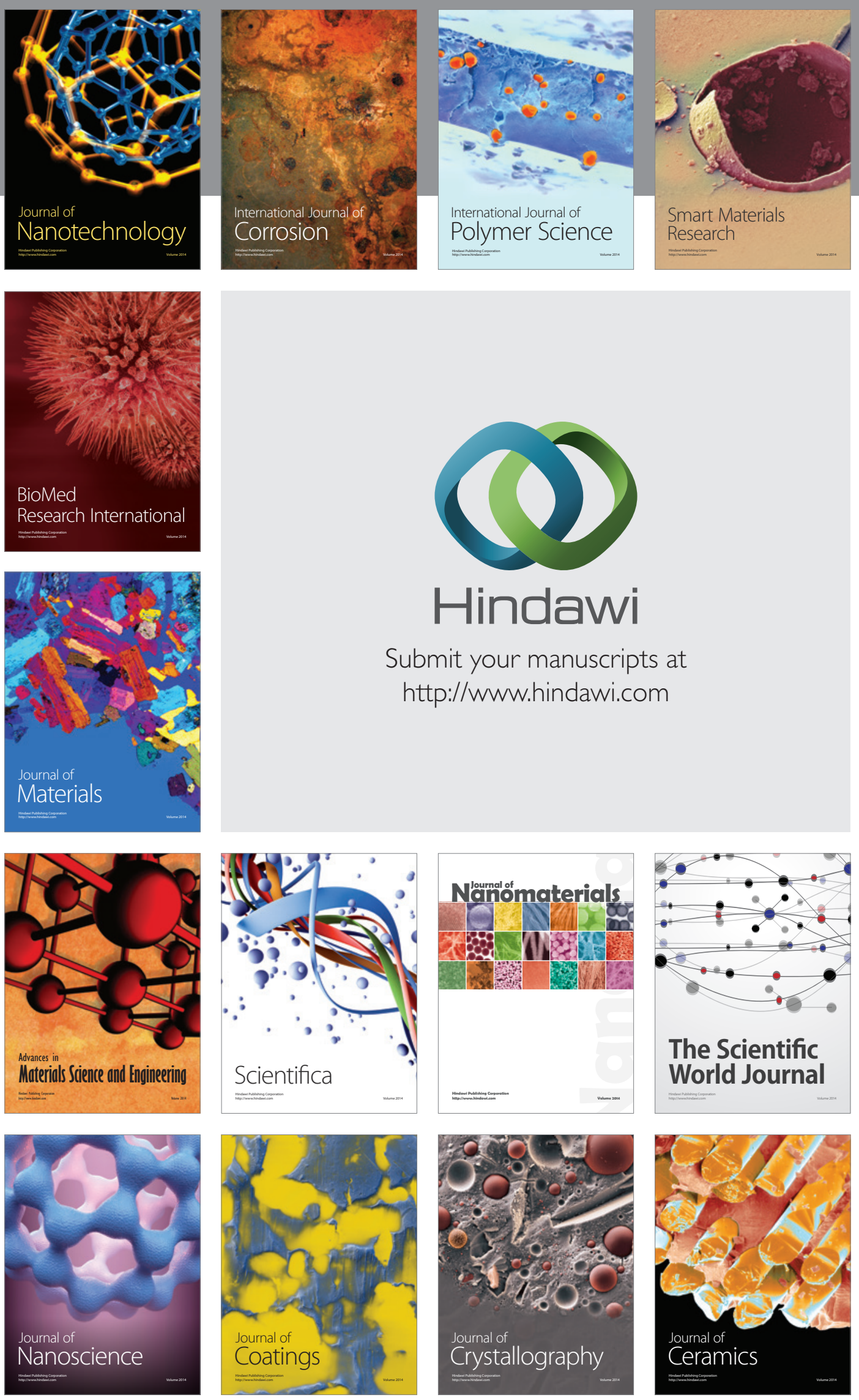

The Scientific World Journal

Submit your manuscripts at

http://www.hindawi.com

\section{World Journal}

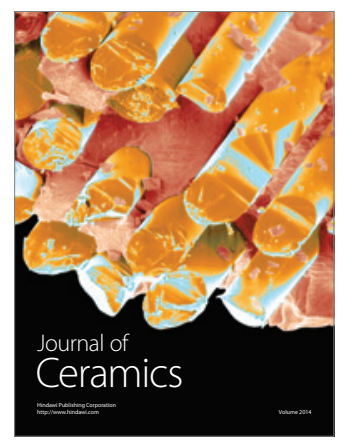

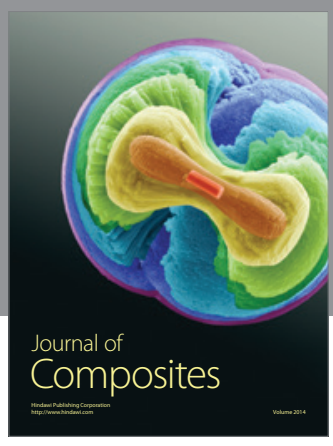
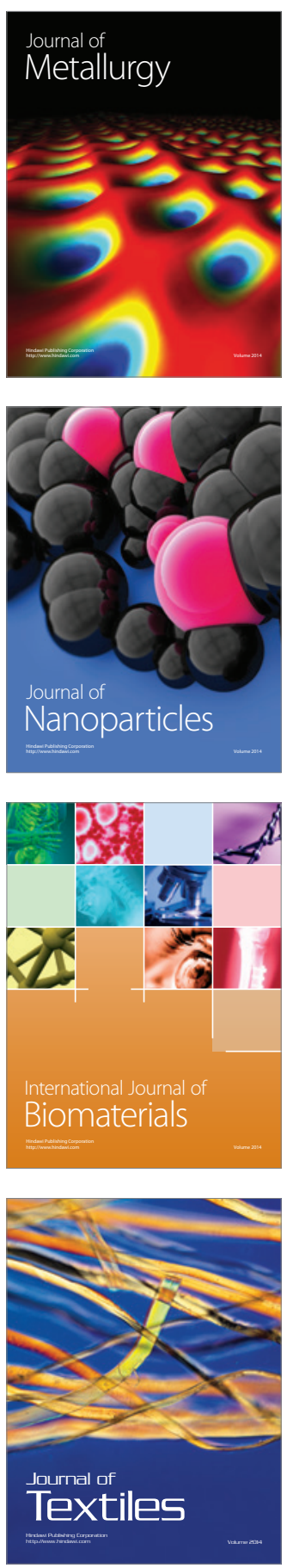